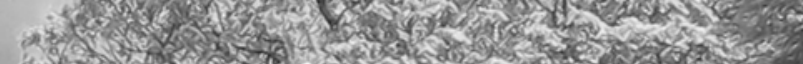

A.t.

- abricis.

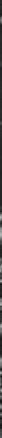

= Min

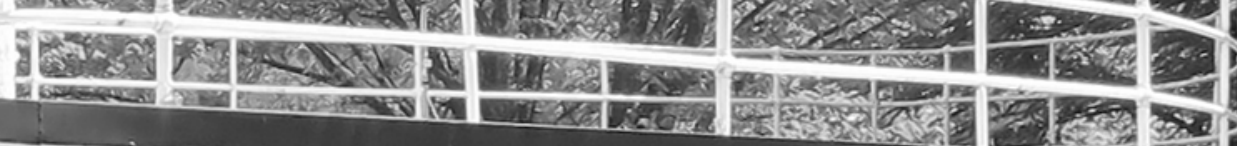

Thes

$-2+\frac{3}{3}$

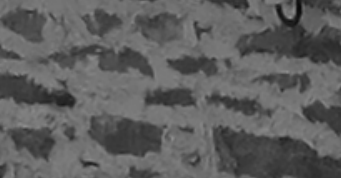

$25 y$
$y-15$

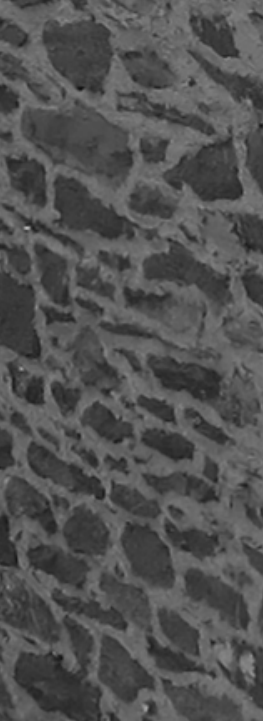
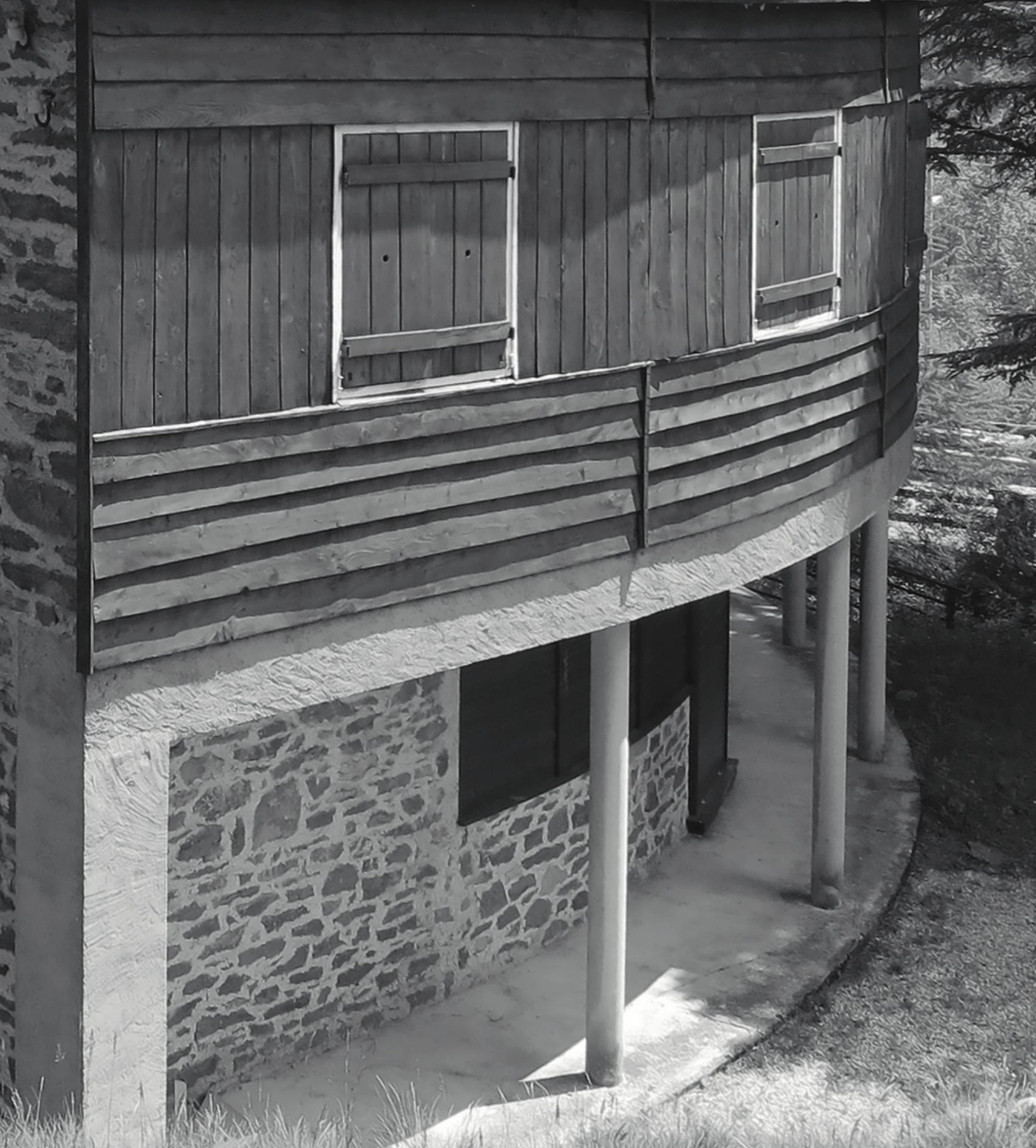


\title{
La "nave" che salpa verso il paesaggio alpino: villa Borsotti a Balme
}

The "ship" that sets sail for the Alpine landscape: Villa Borsotti in Balme

\begin{abstract}
The project for Villa Borsotti, whose construction ended in 1932, is the result of a collaboration between the architect Umberto Cuzzi and the artist Gigi Chessa, who built this small house at the edge of the village of Balme in Val d'Ala di Lanzo, in the area surrounding Turin.

The essay focuses on the genesis of the project, with reference to the cultural and professional context within which the protagonists have worked.

In terms of the relationship between the external aspect and its location in the Alpine context, the building seems to be characterized by the presence of two apparently opposite tendencies.

On the one hand, the building looks for a contextualization in the mountain landscape through the declination in local key of a rationalist language, with a modern use of local dialect, composed of "lemmas" from the Alpine building tradition (stone masonry, wooden infill, bipartition between stone basement and wooden upper floor, etc.). At the same time, thanks to the bending configuration of the plan and the ribbon window, the surrounding environment also "enters" the house and becomes an integral part of it.

On the other, the house seems to pursue the effect of alienation from the context through the conscious research of a formal autonomy with which the object "lands" in the natural framework of the valley.

Another interesting trait of the house is the treatment of interiors according to the idea of configuring a wrap-around environment in which architecture and interior design are strongly intertwined.
\end{abstract}

\section{Roberto Dini}

Architect and PhD at the Politecnico di Torino, where he work as researcher and teacher. He studies the recent transformations of the alpine landscape and territory in the research centre "Istituto di Architettura Montana» (IAM). His publications include Architetture del secondo Novecento in Valle d'Aosta (Regione Autonoma Valle d'Aosta - Mibact, 2018), Rifugi e Bivacchi. Gli imperdibili delle Alpi (Hoepli, 2018), Architecture in the Alps. Heritage and design (Mimesis, 2017), Architettura alpina contemporanea (Priuli \& Verlucca, 2012).
Keywords

Gigi Chessa, Umberto Cuzzi, modernism, rationalism, landscape. 
«Nella villa - denominata dai valligiani "la nave" approdata sui 1500 metri, Cuzzi è riuscito ad esprimersi con quella apparente semplicità che è soltanto dei grandi artisti. Non v'è nulla di gratuito nel piccolo edificio che unisce mirabilmente i materiali locali con i più spinti dettami del razionalismo puro. Fino ad ora inoltre, non si sono verificati inconvenienti funzionali o tecnici, malgrado soluzioni arrischiate come il tetto piano, ecc.». (Pozzetto, 1974).

Con queste parole lo storico dell'architettura Marco Pozzetto, mette in luce alcuni degli aspetti salienti di questo piccolo manufatto: la declinazione in chiave locale del linguaggio razionalista dell'epoca ma allo stesso tempo la volontà di creare un effetto estraniante con la consapevole ricerca di una autonomia formale dell'oggetto architettonico, facendolo "approdare" nel contesto naturale della valle. L'edificio, la cui costruzione termina nel 1932, nasce da una collaborazione tra l'architetto Umberto Cuzzi e l'artista Gigi Chessa che, su incarico della famiglia torinese Borsotti, progettano questa piccola abitazione a monte del centro abitato di Balme in Val d'Ala di Lanzo, in provincia di Torino.

\section{Lo spazio come sintesi tra arte e architettura}

Per comprendere la genesi di questa opera è necessario prima di tutto tracciare un breve racconto che illustri il contesto professionale ma soprattutto culturale all'interno del quale hanno operato gli autori del progetto, le prerogative e la natura della collaborazione tra i due.

Umberto Cuzzi nasce in Istria nel 1891 e termina gli studi a Torino, dove morirà nel 1973. Egli si forma inizialmente a Vienna dove si iscrive alla Technische Hochschule che però abbandona per arruolarsi nell'esercito austroungarico. Dopo la guerra si iscrive al Politecnico di Torino dove conclude gli studi nel 1921. Negli anni successivi torna a lavorare a Gorizia come collaboratore presso lo studio di Silvano Barich, partecipa in seguito ad alcuni concorsi e progetta diversi edifici. zie anche al conterraneo Giuseppe Pagano che vi si era già stabilito - entra in contatto con gli esponenti piemontesi del razionalismo che hanno aderito al
M.I.A.R. (Movimento Italiano Architettura Razionale) tra cui Gino Levi Montalcini, Ettore Sottsass, Ottorino Aloisio.

Con questi darà vita ad un'intensa collaborazione che li vedrà partecipare alla II Esposizione di Roma del M.I.A.R., con un progetto di villa (1931) e al concorso per la ricostruzione della seconda parte di via Roma di Torino (1932). Progetta diverse opere a Torino, come il mercato ortofrutticolo (1933), e dal 1934 fino allo scoppio della seconda

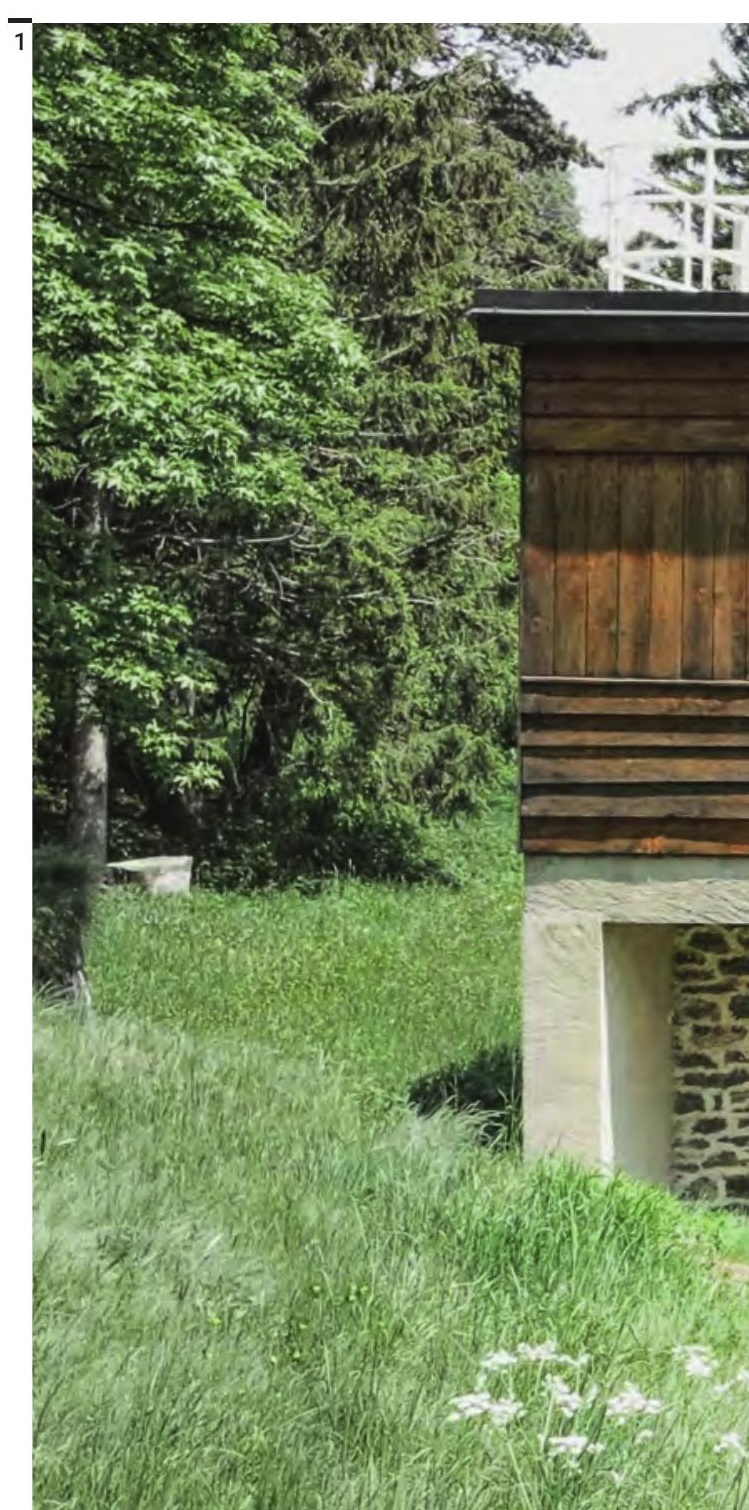


guerra mondiale lavora in modo continuativo con Gino Levi Montalcini, Ettore Sottsass ed Emilio Pifferi con i quali partecipa a concorsi come quelli per il palazzo del Littorio e per la Mostra della Rivoluzione fascista a Roma (1934), per il palazzo della Civiltà del lavoro, il palazzo dei Congressi ed il ministero per l'Africa italiana all'EUR a Roma (1937), per la ricostruzione del teatro Regio di Torino (1938).

Gli interessi di Gigi Chessa spaziano dalla pittura alla scenografia, dall'architettura al teatro, dall'arredamento alla pubblicità. Si forma tra il 1914 e il 1918 all'Accademia Albertina per poi diventare apprendista presso lo studio del pittore Agostino Bosia. A partire dal 1918 espone proprie opere a Torino. Nel 1923 viene premiato alla I Biennale delle arti decorative di Monza, partecipa alla I Quadriennale di Torino e alla XIV Mostra veneziana di Ca' Pesaro, nel 1924 espone alla galleria Pesaro di Milano e al Lido di Venezia, nel 1925 alla Società Fontanesi di Torino.
A partire dal 1928, egli entra far parte del "gruppo dei sei di Torino" che si riuniscono attorno alla figura del maestro Felice Casorati, insieme a Francesco Menzio, Carlo Levi, Jessie Boswell, Enrico Paulucci e Nicola Galante, andando così a costituire uno dei primi sodalizi artistici italiani che guarda oltralpe alle esperienze avanguardistiche in corso in Europa (D’Orsi, 2000).

$\grave{E}$ in questo contesto che, grazie anche all'occasione offerta da alcuni eventi espositivi - si veda ad esempio l'Esposizione Internazionale di Torino del 1928 (per la quale egli realizza il Padiglione dei Fotografi a cui si affianca il meno noto Padiglione delle Valli di Lanzo) - Chessa matura un approccio che, nella concezione dello spazio, guarda ad una sintesi delle arti nell'architettura (Pozzetto, 1974), secondo una sorta di declinazione in chiave locale di alcuni temi cari alle avanguardie internazionali.

Ciò è particolarmente evidente in diverse realizzazioni di ambienti d'interno che si collocano al confi-
Roberto Dini).

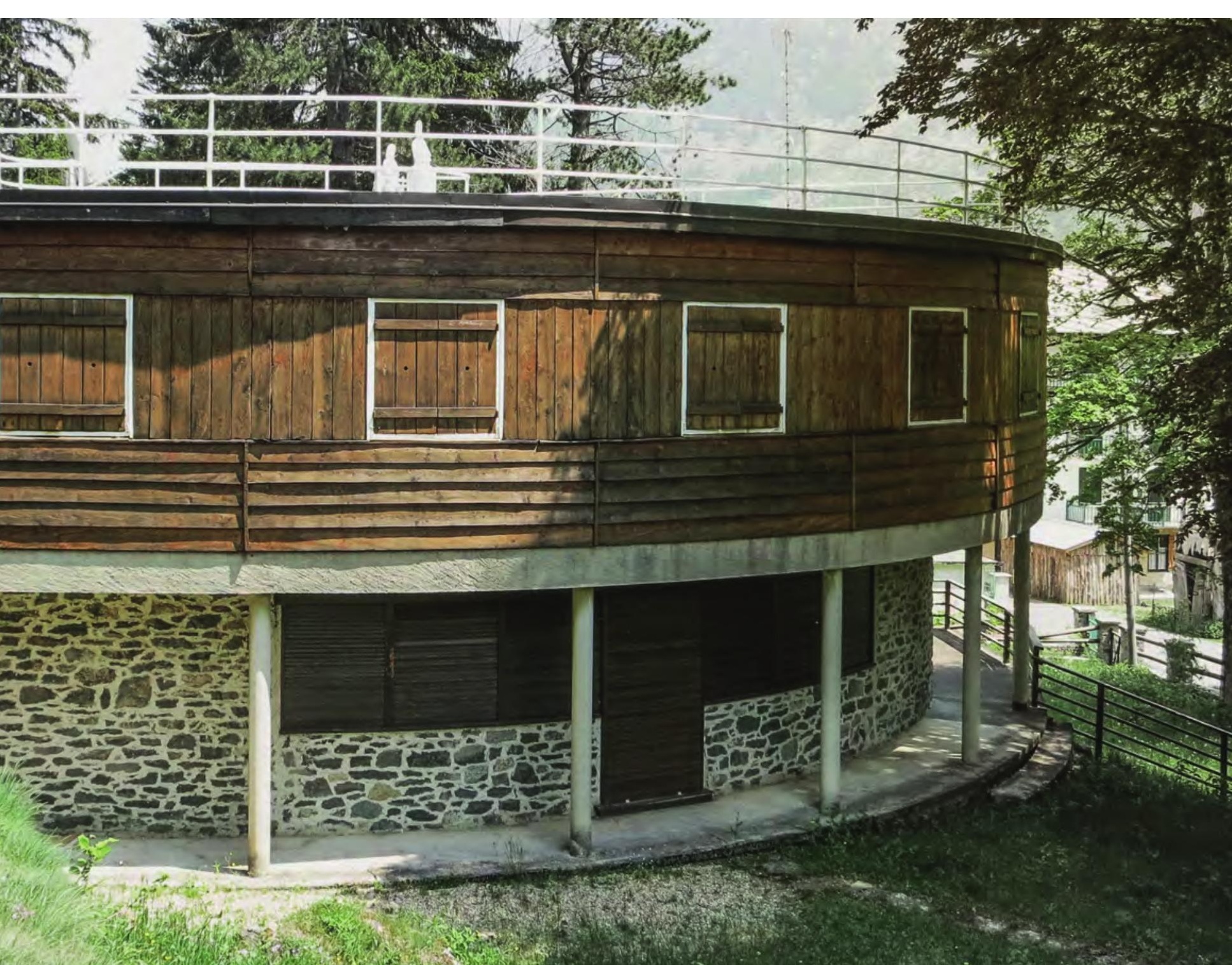




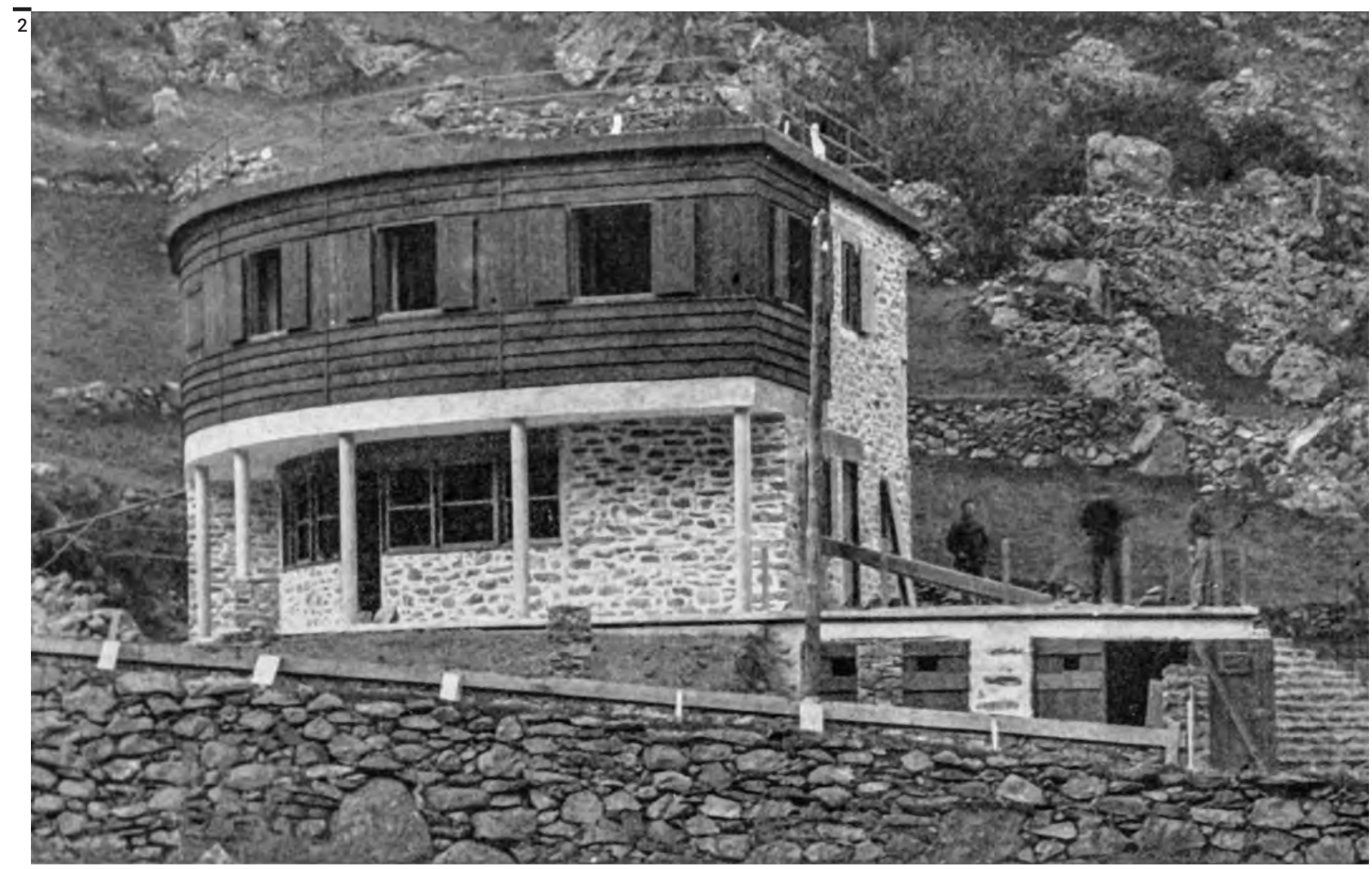

ne tra arredamento e architettura, molte delle quali progettate insieme allo stesso Cuzzi: la sala dei Metalli alla IV Triennale di Monza, anche con Carlo Turina (1930), una sala da musica (1931), uno studio di ufficio per uomo d'affari (1931), alcune vetrine per la Mostra della moda e l'arredamento del caffè Fiorina a Torino (1932), la Sala d'Estate alla V Triennale, con Pagano, Paolucci delle Roncole, Turina e Aloisio (1933).

Con Cuzzi, Chessa comincia dunque una assidua collaborazione fin dal trasferimento a Torino dell'architetto istriano, in un clima di affinità e unione d'intenti in cui è possibile collocare anche il progetto per la villa di Balme: «con Umberto Cuzzi, Gigi Chessa ebbe una collaborazione lunga e assai proficua, che crediamo di poter affermare avvenuta alla insegna della parità e della dialettica tra autonomia e complementarietà culturale di entrambi [...]» (Castagno, Mosso, 1987).

I due lavorano alla creazione di un ambiente architettonico "totale" in cui vi è una forte compenetrazione tra spazio architettonico e arredamento. L'apporto di Chessa al progetto della casa di Balme risulta quindi decisivo nella definizione degli spazi interni che presentano forti analogie con i coevi lavori allestitivi e di arredamento: «fermiamo infine la nostra attenzione sugli ambienti che coronano al più alto livello, il percorso artistico di Gigi Chessa: la villa Borsotti a Balme e il caffè Fiorina, nati in un singolare gemellaggio di concezione e attuazione con richiami reciproci evidenti nei materiali e nei dettagli costruttivi e decorativi» (Castagno, Mosso, 1987).

Come si può notare nel trattamento degli spazi interni della villa, superato il filtro costituito dall'ingresso, è il grande semicerchio convesso su cui si sviluppa la finestra a nastro a caratterizzare lo spazio della sala del soggiorno.

Alle pareti un rivestimento in doghe di legno chiaro rende l'ambiente ancora più luminoso che contrasta con il tono scuro delle porte interne definite anche dai profili neri; in posizione baricentrica è collocato il camino quadrangolare rivestito in intonaco grezzo e scandito da corsi orizzontali in mattoni e sovrastato da uno specchio inserito in uno sfondato.

\section{Il rapporto con l'ambiente alpino}

«La villa Borsotti è una delle rare "architetture di montagna" autenticamente moderne senza citazioni o cedimenti di mimesi formale dell'architettura contadina, che discendono da una lettura superficiale e distorta della storia stessa dell'architettura» (Castagno, Mosso, 1987). 
Fig. 2

Foto del cantiere della villa.

Fig. 3 Dall'alto verso il basso: planimetria generale, pianta del piano terra e del primo piano (La Casabella, n. 52, 1932).

Fig. 4 Il soggiorno (foto Archivio Borsotti). $\overline{3}$

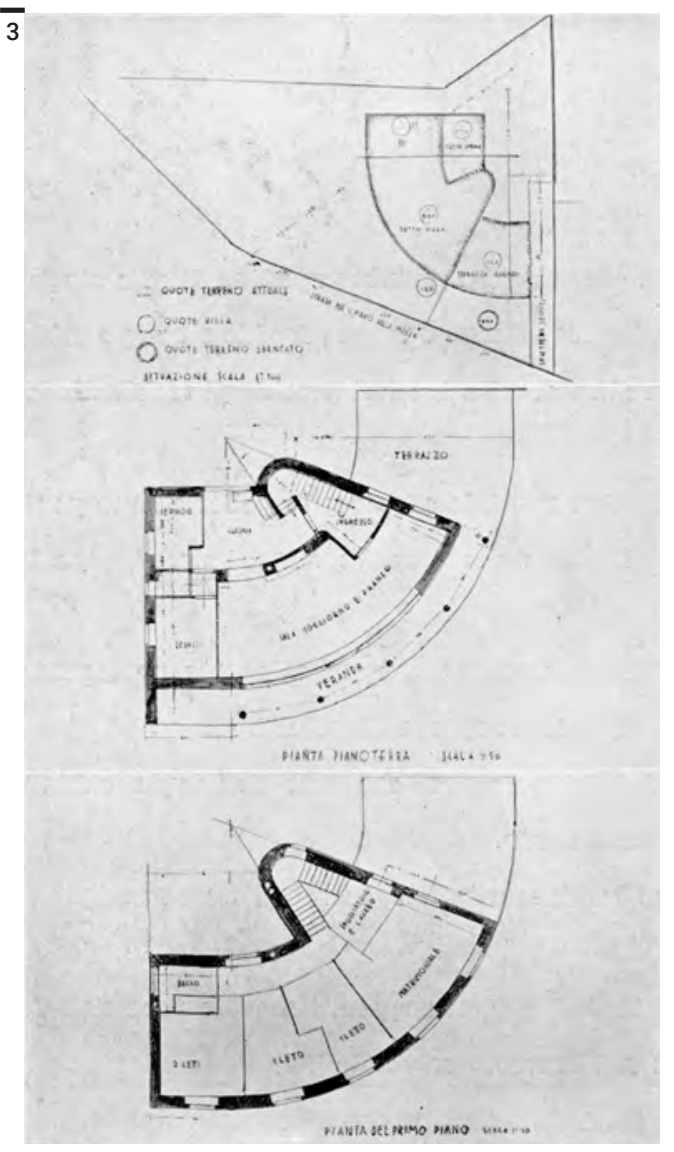

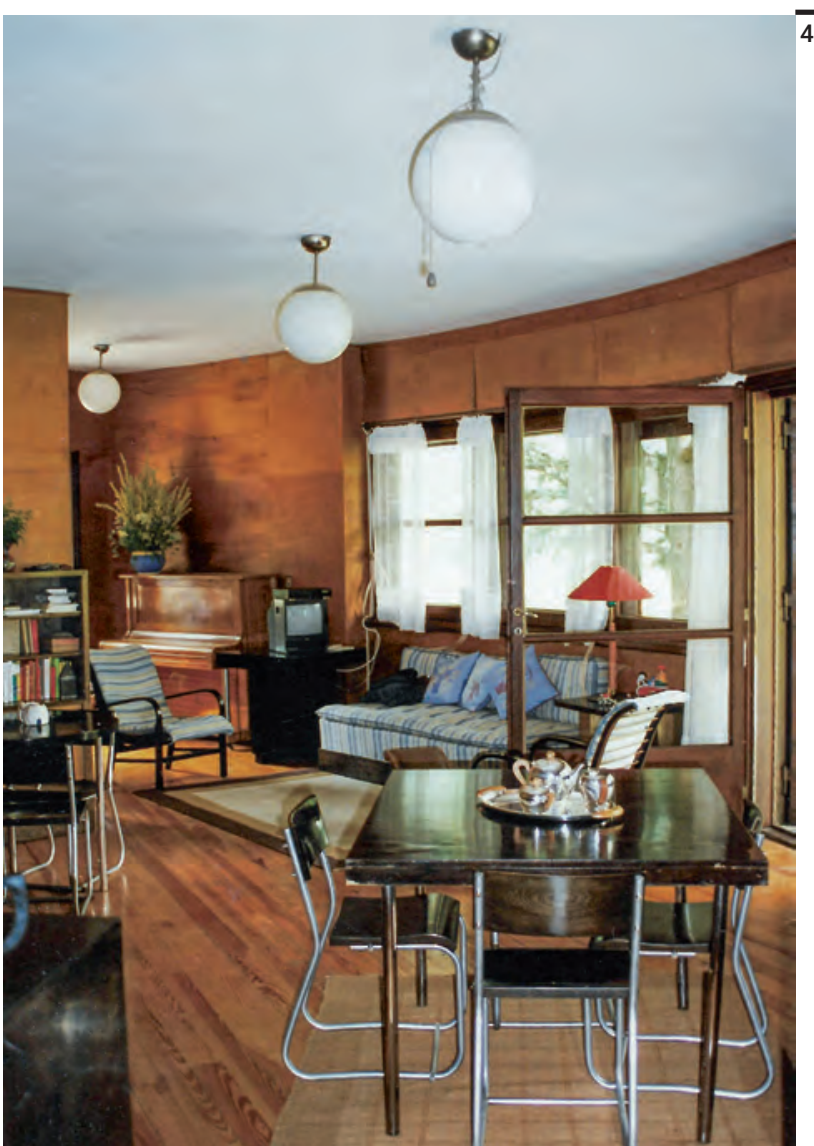



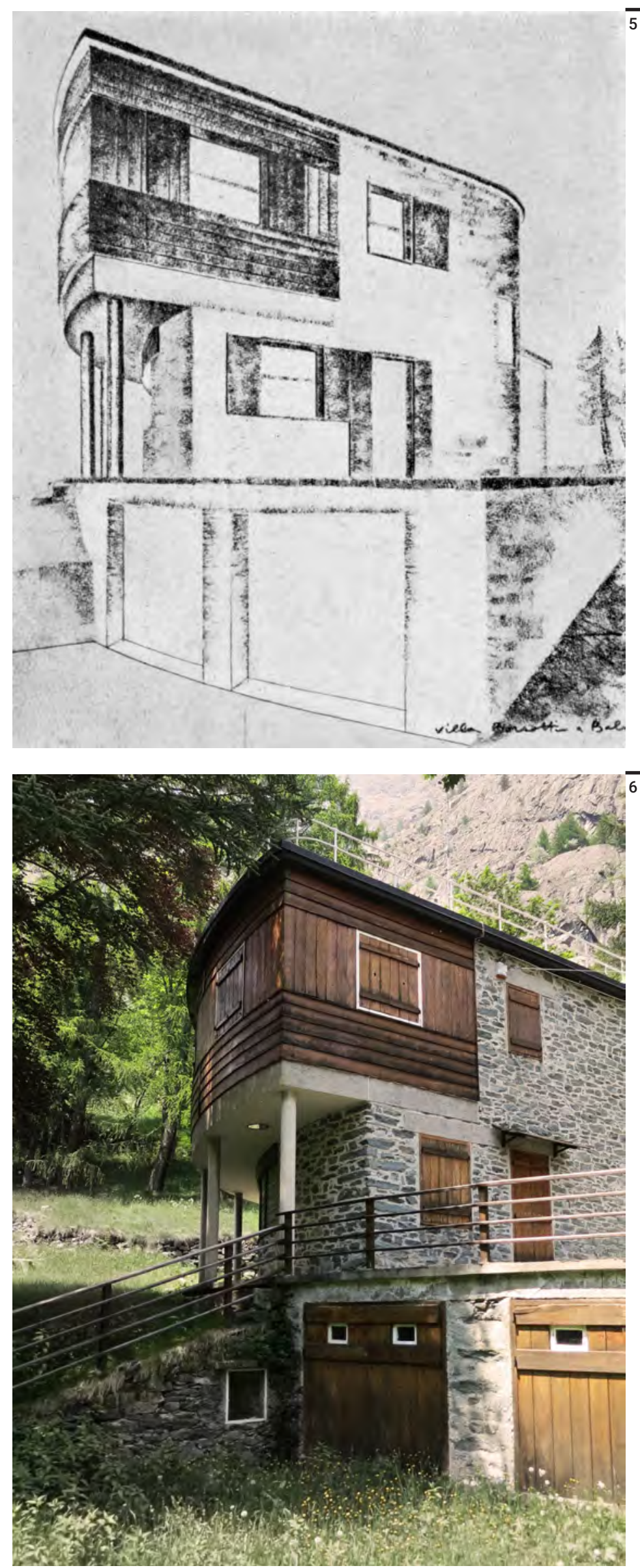

Da ciò può dunque apparire più chiara l'analogia formale con alcuni edifici realizzati in Tirolo da Welzenbacher come la casa Buchroithner a Zell am See (1925-1928), o la casa Rosenbauer a Linz (1929-1930) - entrambe citate nel saggio di De Rossi e Mascino presente in questo numero - o ancora il Turmhotel Seeber ad Hall (1930).

\section{Suggestioni}

In conclusione, ciò che sembra caratterizzare l'aspetto esterno dell'edificio e la sua collocazione in relazione al contesto alpino, è la compresenza di due tensioni apparentemente opposte.

Da un lato abbiamo, come detto, la ricerca di una contestualizzazione dell'edificio nel paesaggio montano. L'uso moderno di un "dialetto locale", fatto con i lemmi della tradizione costruttiva alpina (la muratura in pietra, il tamponamento in legno, la bipartizione tra basamento lapideo e piano superiore ligneo, ecc.), si completa con l'introiezione dell'ambiente circostante che, grazie alla configurazione planivolumetrica ad andamento semicircolare e alla finestra a nastro, "entra" nella casa e ne diventa parte integrante.

Dall'altro lato, all'opposto, la volontà di creare un effetto estraniante con la consapevole definizione di un oggetto dotato di una sua autonomia formale che contrasta con le forme del contesto naturale e culturale della montagna.

L'appellativo di "nave", da parte dei valligiani, non è dunque del tutto errato se - accanto allo scontato significato di oggetto che non risponde ai consueti canoni del "costruire in montagna" - proviamo ad accostare una lettura più attenta che tiene conto della ricerca di nuove suggestioni di carattere compositivo e linguistico che l'architettura moderna pratica in quegli anni.

Pensiamo alle soluzioni formali sviluppate attraverso l'immaginario "navale", come nel caso di diverse colonie marine o abitazioni lungolago degli anni Venti e Trenta che, nella ricerca di una non facile integrazione con il paesaggio naturale marino o lacustre, vengono concepite per contrasto attraverso l'uso di caratteri compositivi ed elementi tipici delle imbarcazioni: chiglia, ponte, prua, poppa, plancia, ecc. diventano così figurazioni in uso anche nell'architettura. Si tratta probabilmente di un tema importato per analogia anche per le costruzioni in quota - si veda ad esempio il progetto per il rifugio/sommergibile Vittorio Emanuele II di Armando Melis - laddove l'incommensurabilità del paesaggio naturale con cui ci si deve confrontare suggerisce di concepire l'edificio come un'entità autonoma che "naviga" nella vastità oceanica dello scenario dell'alta montagna. 


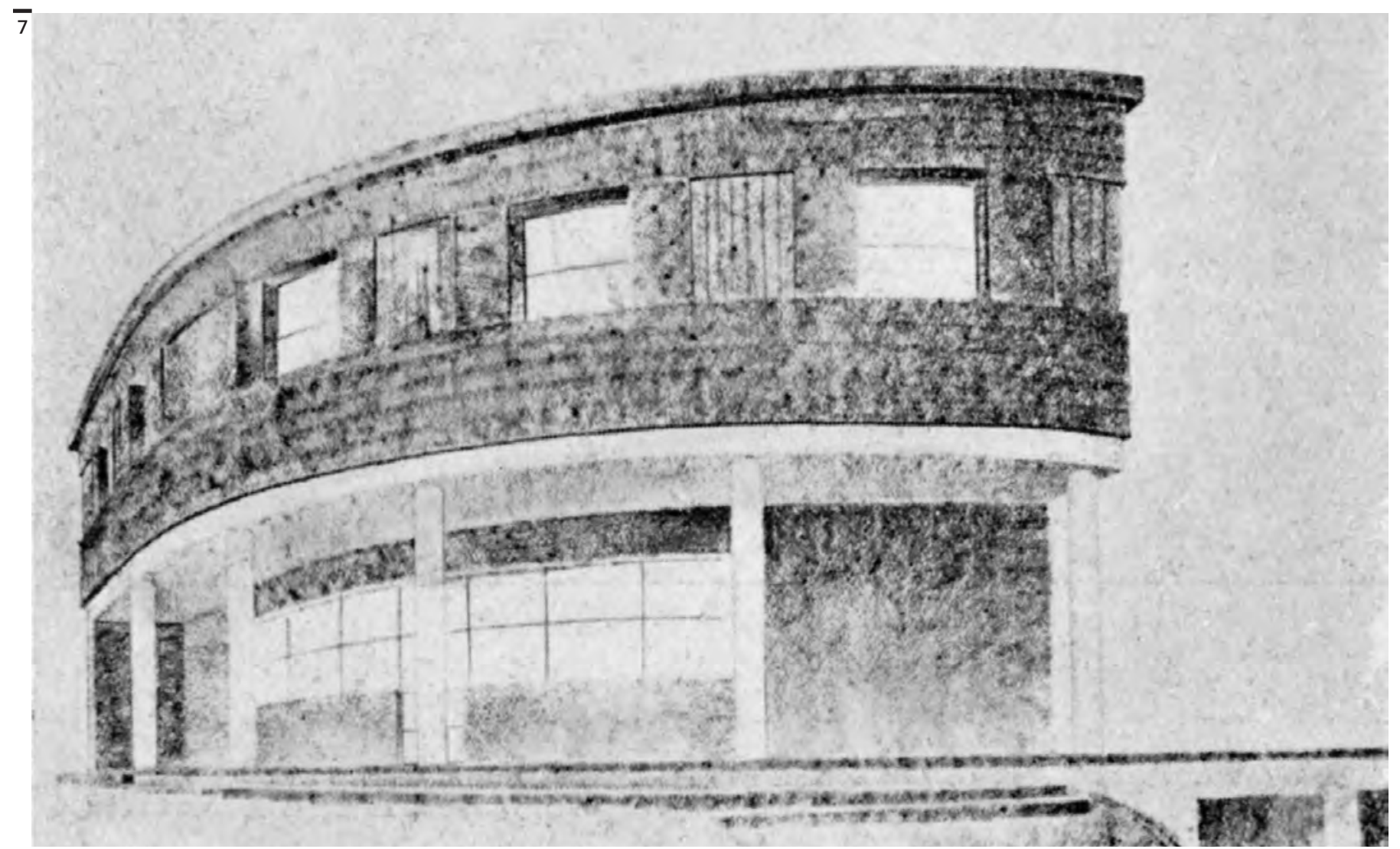

\section{Bibliografia}

Bobbio Norberto (2002), Trent'anni di storia della cultura a Torino (1920-1950), Einaudi, Torino, (I ed. CRT, 1977).

Castagno Laura, Mosso Leonardo (1987), «La ricerca in architettura di Gigi Chessa», in AA.VV., Gigi Chessa 18981935, catalogo della mostra (Torino, Mole Antonelliana, 14 novembre 1987-14 febbraio 1988), Fabbri, Torino, pp. 38-49.

Cennamo Michele (a cura di) (1976), Materiali per l'analisi dell'architettura moderna il MIAR, Società Editrice Napoletana, Napoli.

D’Orsi Angelo (2000), La cultura a Torino tra le due guerre, Einaudi, Torino.

Levi Montalcini Gino (1932), «Piccole ville: 3. In Montagna», in La Casabella, n. 52, p. 20.

Figg. 5 e 7 Levi Montalcini Gino (1934), Umberto Cuzzi architetto, Hermes, Merano.

Disegni Levi Montalcini Gino (1936), «Gigi Chessa 1898-1935», in Domus, n. 104, pp. 3-7.

(La Casabella, n. 52,

Magnaghi Agostino, Monge Mariolina, Re Luciano (1982), Guida all'architettura moderna di Torino, Lindau, Torino.

Pagano Giuseppe (1932), «Quattro progetti di piccoli alberghi di montagna», in Domus, n. 51, pp. 395-400.

1932). Persico Edoardo (1935), "Gigi Chessa», in La Casabella, n. 89, pp. 2-3.

Pozzetto Marco (1974), «Umberto Cuzzi, architetto: equilibrio d'un gusto», in Iniziativa Isontina, XVI, pp. 29-36.

Pozzetto Marco (1975), «Gino Levi Montalcini (1902-1974)», in Studi Piemontesi, vol. IV, p. 136.

Fig. 6 Pozzetto Marco (2008), Figure della Mitteleuropa e altri scritti d'arte e di architettura, Zandonai, Rovereto.

Stato attuale Signorelli Bruno (1985), «Umberto Cuzzi», in Dizionario Biografico degli Italiani, vol. 31, Istituto della Enciclopedia (foto Roberto Dini). italiana, Roma. 